\title{
阻变存储器无源高密度交叉阵列研究进展
}

\author{
李晓燕 ${ }^{1,2}$, 李颖㢷 ${ }^{2 *}$, 高晓平 ${ }^{1}$, 陈传兵 ${ }^{2}$, 韩根亮 ${ }^{1 *}$
}

1. 甘肃省传感器与传感技术重点实验室, 兰州 730000;

2. 兰州大学物理科学与技术学院, 兰州 730000

* 联系人, E-mail: li_yt06@1zu.edu.cn; genlianghan@163.com

2018-03-09 收稿, 2018-04-22 修回, 2018-04-23 接受, 2018-09-27 网络版发表

国家自然科学基金(61774079, 61664001)、甘肃省科技重点研发计划(18YF1GA088)和甘肃省自然科学基金(17JR5RA180)资助

\begin{abstract}
摘要阻变存储器(resistive random access memory, RRAM) 以其结构简单、操作速度快、可缩小性好、易三维(3D) 集成、与互补金属氧化物半导体(complementary metal oxide semiconductor, CMOS)工艺兼容等优势成为下一代非 挥发性存储器的有力竞争者之一, 但基于阻变存储器无源交叉阵列中的交叉串扰问题影响了其实现高密度存储的 应用和发展。本文简单介绍了阻变存储器交叉阵列中的串扰现象, 详细综述了避免无源交叉阵列串扰的1D1R(one diode one resistor)结构、1S1R(one selector one resistor)结构、背靠背(back to back)结构及具有自整流效应的1R(one resistor)结构. 同时, 对基于阻变存储器无源交叉阵列实现高密度存储的研究发展趋势以及面临的挑战进行了展望.
\end{abstract}

关键词阻变存储器, 高密度, 无源交叉阵列, 交叉串扰

微电子技术是当代高科技和信息产业的核心技 术，在国民经济、国防建设以及信息化社会中有着极 其重要的战略意义, 集成电路从 20 世纪 60 年代的“小 规模集成”发展到现在的“超大规模集成”. 而作为微 电子技术的重要组成部分, 半导体存储器技术也迅 速朝着“更高密度、更快速度、更低功耗”的方向发展. 特别是近年来, 随着移动智能终端、云计算、物联网、 大数据等新信息技术的快速发展与普及, 消费市场 对于非挥发性存储器的需求越来越大. 基于电荷存 储机制的非挥发性浮栅存储器(flash), 作为目前非挥 发性半导体存储器市场中的主流产品和代表性技术, 由于其编程电压较高、读写速度较慢、功耗较大及隧 穿氧化层厚度减小会引起漏电流增加等问题，随着 半导体技术工艺节点的不断提升, Flash存储器在可 缩小性、功耗、可靠性等方面遇到的技术瓶颈更加严 峻 $^{[1,2]}$. 近年来半导体业界、科研界和学术界, 采用基 于电阻值变化作为信息存储方式, 实现了以磁存储
器( magnetic random access memory, MRAM)、相变存 储器(phase-change random access memory, PRAM)和 阻变存储器(resistive random access memory, RRAM) 等为代表的新型非挥发性存储器 ${ }^{[3 \sim 5]}$. 其中, 阻变存 储器具有可缩小性好、功耗低、操作速度快、非破坏 性读取以及与CMOS工艺兼容等优点, 因此备受半导 体产业界的关注 ${ }^{[6,7]}$.

阻变存储作为一种新兴存储技术, 能否实现高 密度存储是其立足的根本. 因此, 高密度集成成为近 年来RRAM研究的重点. RRAM器件因为能够将单元 面积做到 $4 \mathrm{~F}^{2}(\mathrm{~F}$ 为每个工艺技术代的特征线宽), 这是 理想的最小存储单元面积. 因此, 基于RRAM器件的 交叉阵列被认为是实现高密度存储最简单、最有效的 方法. 基于 RRAM的交叉阵列集成一般分为有源阵 列 (active)和无源阵列 (passive)两种, 有源阵列的基本 单元是一个晶体管作为选择管串联一个阻变单元构 成的1T1R(one transistor one resistor)结构, 无源阵列

引用格式: 李晓燕, 李颖㢷, 高晓平, 等. 阻变存储器无源高密度交叉阵列研究进展. 科学通报, 2018, 63: 2954-2966

Li X Y, Li Y T, Gao X P, et al. An overview of resistive random access memory based high-density crossbar array (in Chinese). Chin Sci Bull, 2018, 63: 2954-2966, doi: 10.1360/N972018-00164 
基本单元主要包括1D1R结构、1S1R结构和1R结构. 有源阵列中每个存储单元的最小面积由晶体管的大 小决定, 最小单元面积为 $6 \mathrm{~F}^{2}$, 这就丧失了 RRAM可 缩小性的优势. 再者, $1 \mathrm{~T} 1 \mathrm{R}$ 结构中 $\mathrm{T}$ 的制备是在前段 工艺完成, 需要高温制备过程, 不能够实现三维(3D) 集成, 因此在高密度集成上存在劣势. 而无源交叉阵 列结构则工艺制备简单, 同时可以采用三维的多层 集成, 实现高密度存储, 被认为是目前存储器件最经 济的集成方式 ${ }^{[8]}$.

本文首先介绍了基于有源阵列的1T1R结构以及 无源交叉阵列中的串扰现象. 其次, 对解决无源交叉 阵列串扰问题的1D1R结构、1S1R结构、背靠背结构, 以及具有自整流效应的 $1 R$ 结构进行了总结. 最后对 RRAM无源交叉阵列的高密度集成目前存在的主要 问题以及研究的重点作了简单评述.

\section{1 阻变存储器的集成}

阻变存储器的集成通常分为有源阵列和无源阵 列, 有源阵列一般是由场效应晶体管(MOSFET)和阻 变存储单元串联构成1T1R结构, 其中晶体管起到选 通和隔离的作用, 即当对阻变存储单元进行操作时, 选择的晶体管导通, 选择所需的操作单元, 而其他阻 变存储单元的晶体管则关闭, 这样能避免对周围单 元产生串扰和误操作, 从而起到隔离的作用, 并在集 成阵列中利用字线和位线来达到选通存储单元的目 的 $^{[9 \sim 12]}$. 有源阵列的制作, 晶体管通常在前端工艺完 成, 阻变存储器则在后端工艺完成, 所以工艺集成必 须考虑热预算, 且制备过程中的温度不能过高. 阵列 中晶体管的大小决定了每个存储单元的面积, 假定 在特定的技术节点下最小选择晶体管能提供足够大 的驱动电流使阻变存储单元发生电阻转变, 每个存 储单元的面积则为 $6 \mathrm{~F}^{2[10]}$.

对于无源阵列, 每个存储单元是由相互交叉的 字线(word line, WL)和位线 (bit line, BL)构成的上下 电极所确定, 平面结构能够实现最小的存储单元面 积一 $-4 \mathrm{~F}^{2}$. 无源交叉阵列的制备工艺相对简单, 通 常是将CMOS电路在前端工艺完成, RRAM则在后端 工艺中完成, 由于无源阵列不依赖CMOS工艺的前端 制程, 因此能够进行多层堆叠, 实现三维存储结构, 所以每个存储单元最小面积为 $4 \mathrm{~F}^{2} / \mathrm{N}(\mathrm{N}$ 为堆叠的层 数), 存储密度可以成倍提高 ${ }^{[13]}$, 相比无源阵列, 有 源阵列由于单元面积大, 需占用衬底硅的面积, 故而
不利于三维集成. 因此, 综合考虑存储阵列集成密度 和工艺这两方面的因素, 无源交叉阵列才是RRAM 集成的首选方式, 这也被认为是目前存储器件最经 济的集成方式 ${ }^{[8]}$.

但是由于构成无源阵列结构RRAM的低阻态通 常表现为欧姆特性, 在读取相邻交叉节点的阻值时 易产生串扰(cross-talk)现象, 这样就会导致误读, 随 着存储阵列堆叠层数越多, 误读现象会变得更加严 重, 因此在采用无源交叉阵列时, 要使每个交叉点具 有整流特性, 以此来避免交叉串扰引起的误读现象.

\section{2 无源交叉阵列中的串扰现象}

如图 1所示的交叉阵列, 以一个最简单 $2 \times 2$ 的阵 列结构为例, 如果有一个RRAM器件单元 $A$ 处于高阻 态而其他 3 个 RRAM器件单元B, C, D处于低阻态, 在 读取单元 $A$ 的状态时电流将沿着 3 个处于低阻态的器 件单元形成一条漏电通道, 如图1中的虚线所示. 形 成的漏电通道不光增加不必要的功耗, 而且使得读 出来的电阻值不是器件单元 $\mathrm{A}$ 的真实电阻值, 这就是 交叉阵列的串扰问题, 即所谓的误读现象 ${ }^{[14 \sim 17]}$. 当 阵列变大或者多层阵列堆叠时, 所述漏电通道将随 之增多, 增大的漏电流使得误读现象更为严重. 为解 决RRAM交叉阵列中的交叉串扰问题, 需要存储单 元具有整流特性, 通常在每个阻变存储单元处串联 一个具有整流特性的选择单元来构成新的器件结构. 目前解决RRAM无源交叉阵列中串扰问题的主要器 件结构包括: 1D1R结构、1S1R结构、back to back结构, 以及具有自整流效应的 $1 \mathrm{R}$ 结构.

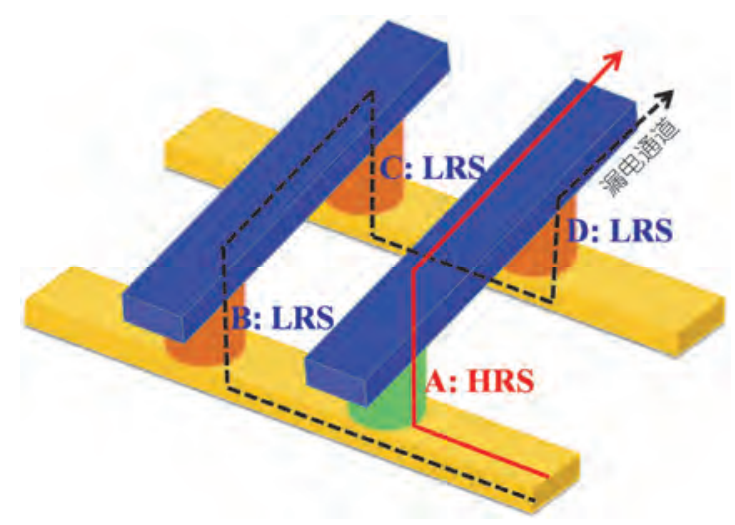

图 1 (网络版彩色)无源交叉阵列的串扰示意图

Figure 1 (Color online) Crosstalk schematic diagram of passive cross array 


\section{1D1R结构}

基于1D1R结构的无源交叉阵列, 是在每个阻变 存储单元上串联一个二极管, 使每个存储单元都有 整流特性, 这不仅不会影响到阵列的存储密度, 而且 能够有效避免交叉阵列中的交叉串扰现象. 由于每 个阻变存储单元都与二极管相连, 即使需要读取的 器件处于高阻状态, 而其余 3 个相邻器件皆处于低阻 状态, 由于二极管具有整流作用, 在需要读取的器件 上加读电压时, 电流也主要通过该器件本身来进行 传导，因而能够抑制“误读”现象.

根据二极管制备材料的不同，目前应用于 $1 \mathrm{D} 1 \mathrm{R}$ 结构中的二极管的类型主要分为基于硅材料的二极 管 ${ }^{[18]}$ 、基于氧化物材料的二极管 ${ }^{[19,20]}$ 、以及基于聚合 物材料的二极管 ${ }^{[21,22]}$. Lu等人 ${ }^{[23]}$ 制备的肖特基型 $\mathrm{Ti} / \mathrm{n}-\mathrm{Si}(001)$ 二极管, 将其与双极性 $\mathrm{Pt} / \mathrm{HfO}_{2} / \mathrm{Ti}$ 结构的 RRAM存储器件单元串联组成交叉阵列后, 器件具 有电阻转变特性, 并且由于二极管的整流效应, 存储 单元在低阻态时具有明显的整流特性, 在 $\pm 2 \mathrm{~V}$ 的读 取电压下整流比可以达到 $10^{3}$, 很好地解决了串扰问 题. 虽然基于 $\mathrm{Si}$ 材料的二极管的电流密度和整流比 相对较高, 但是 $\mathrm{Si}$ 不容易在金属材料上外延得到质 量好的单晶 $\mathrm{Si}$ 或多晶 $\mathrm{Si}$, 制备需要的热处理高温度会 预先破坏淀积层且会在金属层上产生额外的硅化物, 所以不宜用于RRAM在后端制程中的集成 ${ }^{[19]}$. 而基
于氧化物材料的二极管可以在低温甚至在室温下制 备, 并且能够与CMOS工艺兼容, 因此学术界和工业 界更多地致力于研究具有低制备温度、与CMOS工艺 兼容性良好的金属氧化物二极管. 表 1 给出了部分目 前研究报道的可用作RRAM无源交叉阵列选通管的 氧化物二极管器件性能参数.

Lee等人 ${ }^{[26]}$ 在低温下制备了 $\mathrm{p}-\mathrm{NiO}_{x} / \mathrm{n}-\mathrm{TiO}_{x}$ 二极 管, 其整流比在 $\pm 2 \mathrm{~V}$ 读取电压下可达到 $10^{5}$, 且电流 密度为 $5 \times 10^{3} \mathrm{~A} / \mathrm{cm}^{2}$. 将此二极管与双极性阻变存储 单元 $\mathrm{Pt} / \mathrm{NiO} / \mathrm{Pt}$ 串联构成1D1R结构 $\left(\mathrm{Pt} / \mathrm{NiO} / \mathrm{Pt} / \mathrm{p}-\mathrm{NiO}_{x} / \mathrm{n}\right.$ $\left.\mathrm{TiO}_{x} / \mathrm{Pt}\right)$. 如图2所示, 由于串联了二极管, 使得阻变 存储单元在低阻态时具有明显的整流特性, 从而能 够有效抑制串扰现象. Huang等人 ${ }^{[30]}$ 在室温下用电子 束蒸发、等离子增强化学气相淀积等技术制备了金属介质层-金属(MIM)结构的肖特基型 $\mathrm{Ti} / \mathrm{TiO}_{2} / \mathrm{Pt}$ 二极 管, 其整流比在 $\pm 3 \mathrm{~V}$ 读取电压下可达到 $10^{5}$, 正向电 流密度为 $2 \times 10^{3} \mathrm{~A} / \mathrm{cm}^{2}$, 整流特性在 $125^{\circ} \mathrm{C} 、 \pm 3 \mathrm{~V}$ 扫描 模式下能够稳定循环 $10^{3}$ 个周期. 研究表明通过控制 电压开启过程和界面势垒，可以有效改善整流二极 管的特性参数, 减小界面势垒以及减小氧化物半导 体的禁带宽度有助于增加电流密度, 这对提高氧化 物整流二极管的电流密度具有一定的指导意义. Teo 等人 ${ }^{[21]}$ 将制备出的聚合物二极管ITO/PEDOT/P ${ }_{3} \mathrm{HT}$ ： $\mathrm{PCBM} / \mathrm{Al}$ 与非整流的阻变存储器件ITO/PCz/Al串联构

表 1 部分报道的可用于 1D1R集成的整流二极管

Table 1 Comparison of rectifying diodes for 1D1R integration

\begin{tabular}{|c|c|c|c|c|}
\hline 二极管类型 & 器件结构 & 电流密度 & 整流比 & 温度 \\
\hline \multirow[t]{3}{*}{ 硅二极管 } & $\mathrm{p}-\mathrm{Si} / \mathrm{n}-\mathrm{Si}^{[18]}$ & $>10^{3} \mathrm{~A} / \mathrm{cm}^{2}$ & $>10^{5} @ 1 \mathrm{~V}$ & $>1000^{\circ} \mathrm{C}$ \\
\hline & $\mathrm{Ti} / \mathrm{n}-\mathrm{Si}(001)^{[23]}$ & - & $10^{3} @ \pm 2 \mathrm{~V}$ & - \\
\hline & $\mathrm{Al} / \mathrm{p}-\mathrm{Si}^{[24]}$ & - & $10^{4} @ 2.3 \mathrm{~V}$ & - \\
\hline \multirow{5}{*}{$\begin{array}{l}\text { 氧化物二极管 } \\
(\mathrm{PN} \text { 结型) }\end{array}$} & $\mathrm{Pt} / \mathrm{TiO}_{x} / \mathrm{Pt}^{[20]}$ & $50 \mathrm{~A} / \mathrm{cm}^{2} @-1 \mathrm{~V}$ & $10^{3} @ \pm 1 \mathrm{~V}$ & $200^{\circ} \mathrm{C}$ \\
\hline & $(\mathrm{In}, \mathrm{Sn})_{2} \mathrm{O}_{3} / \mathrm{TiO}_{2} / \mathrm{Pt}^{[25]}$ & $400 \mathrm{~A} / \mathrm{cm}^{2} @ 1 \mathrm{~V}$ & $1.6 \times 10^{4} @ \pm 1 \mathrm{~V}$ & $250^{\circ} \mathrm{C}$ \\
\hline & $\mathrm{p}-\mathrm{NiO}_{x} / \mathrm{n}-\mathrm{TiO}_{x}^{[26]}$ & $5 \times 10^{3} \mathrm{~A} / \mathrm{cm}^{2} @ 3 \mathrm{~V}$ & $10^{5} @ \pm 3 \mathrm{~V}$ & $<300^{\circ} \mathrm{C}$ \\
\hline & $\mathrm{p}-\mathrm{NiO}_{x} / \mathrm{n}-\mathrm{ITO}_{x}^{[27]}$ & $>10^{4} \mathrm{~A} / \mathrm{cm}^{2} @ 1.5 \mathrm{~V}$ & - & 室温 \\
\hline & $\mathrm{p}-\mathrm{CuO} / \mathrm{n}-\mathrm{InZnO}{ }_{x}^{[28]}$ & $3.5 \times 10^{4} \mathrm{~A} / \mathrm{cm}^{2} @ 2.45 \mathrm{~V}$ & $10^{6} @ \pm 2.45 \mathrm{~V}$ & 室温 \\
\hline \multirow{3}{*}{$\begin{array}{l}\text { 氧化物二极管 } \\
\text { (肖特基结型) }\end{array}$} & $\mathrm{Ag} / \mathrm{ZnO} / \mathrm{Ti} / \mathrm{Au}^{[29]}$ & $10^{4} \mathrm{~A} / \mathrm{cm}^{2}$ & $>10^{7}$ & $100^{\circ} \mathrm{C}$ \\
\hline & $\mathrm{Ti} / \mathrm{TiO}_{2} / \mathrm{Pt}^{[30]}$ & $2 \times 10^{3} \mathrm{~A} / \mathrm{cm}^{2} @ 3 \mathrm{~V}$ & $10^{5} @ \pm 3 \mathrm{~V}$ & 室温 \\
\hline & $\mathrm{Pt} / \mathrm{TiO}_{2} / \mathrm{Ti}^{[31]}$ & $3 \times 10^{5} \mathrm{~A} / \mathrm{cm}^{2} @ 1 \mathrm{~V}$ & $10^{9} @ \pm 1 \mathrm{~V}$ & $100^{\circ} \mathrm{C}$ \\
\hline \multirow[t]{4}{*}{ 有机二极管 } & $\begin{array}{l}\text { Al/P3HT:PCBM/PED } \\
\text { OT/ITO }^{[21]}\end{array}$ & - & $10^{3} @ \pm 1 \mathrm{~V}$ & - \\
\hline & $\mathrm{P} 3 \mathrm{HT} / \mathrm{n}-\mathrm{ZnO}^{[22]}$ & $10^{4} \mathrm{~A} / \mathrm{cm}^{2} @ 4 \mathrm{~V}$ & $10^{5} @ \pm 4 \mathrm{~V}$ & - \\
\hline & $\mathrm{Au} / \mathrm{P} 3 \mathrm{HT} / \mathrm{PVP} / \mathrm{Al}^{[32]}$ & - & $1 \times 10^{2}-2 \times 10^{3}$ & $60^{\circ} \mathrm{C}$ \\
\hline & $\mathrm{Au} / \mathrm{OPV} 5 / \mathrm{Ti}^{[32]}$ & $0.3 \sim 0.8 \mathrm{~A} / \mathrm{cm}^{2}$ & $10^{2} @ 4 \mathrm{~V}$ & $40^{\circ} \mathrm{C}$ \\
\hline
\end{tabular}



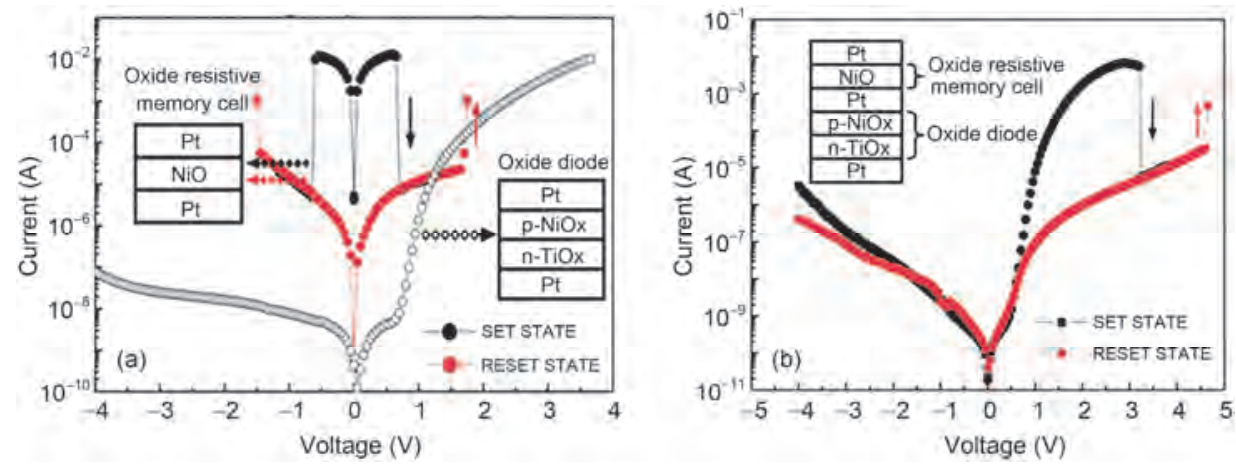

图 2 (网络版彩色) Pt/NiO/Pt/p-NiOx/n-NiOx/Pt1D1R结构的电流电压特性. (a) 阻变存储单元 Pt/NiO/Pt, 二极管 $\mathrm{Pt} / \mathrm{p}-\mathrm{NiO}_{x} / \mathrm{n}-\mathrm{NiO} / \mathrm{Pt}$ 的结构示意 图及 $I-V$ 特性; (b) $\mathrm{Pt} / \mathrm{NiO} / \mathrm{Pt} / \mathrm{p}-\mathrm{NiO}_{x} / \mathrm{n}-\mathrm{NiO}_{x} / \mathrm{Pt} 1 \mathrm{D} 1 \mathrm{R}$ 结构的 $I-V$ 特性曲线 ${ }^{[26]}$

Figure 2 (Color online) $I-V$ characteristics of $\mathrm{Pt} / \mathrm{NiO} / \mathrm{Pt} / \mathrm{p}-\mathrm{NiOx} / \mathrm{n}-\mathrm{NiOx} / \mathrm{Pt} 1 \mathrm{D} 1 \mathrm{R}$ structure. (a) $I-V$ characteristic of a Pt/NiO/Pt device structure, and the rectifying characteristic of a Pt/p-NiO$x / \mathrm{n}-\mathrm{NiO}_{x} / \mathrm{Pt}$ diode structure. The insets show schematic diagrams of Pt/NiO$/ \mathrm{Pt}$ and $\mathrm{Pt} / \mathrm{p}-\mathrm{NiO} x_{x} / \mathrm{n}-\mathrm{NiO} x_{x} / \mathrm{Pt}$ structures; (b) $I-V$ characterstics of a combined $\mathrm{Pt} / \mathrm{NiO} / \mathrm{Pt} / \mathrm{p}-\mathrm{NiO}_{x} / \mathrm{n}-\mathrm{NiO}_{x} / \mathrm{Pt} 1 \mathrm{D} 1 \mathrm{R}$ structure, the schematic diagram is shown in the inset ${ }^{[26]}$

成一次编程(write once read many times, WORM)存储 器, 其整流比达到 $10^{3}( \pm 1 \mathrm{~V}$ 的读取电压), 具有明显 的整流特性, 有效抑制了交叉串扰, 在很大程度上消 除了误读现象. 这种具有整流特性的一次编程存储 器的实现为打开聚合物交叉阵列三维大规模集成之 路提供了很大可能性 ${ }^{[21]}$.

由于二极管的单向导通性，传统的1D1R结构只 适用于单极性阻变存储器. 然而, 从大量的研究报道 中可以发现双极性阻变存储器的性能要明显优于单 极性阻变存储器; 另外, 与晶体管不同, 二极管正向 导通后不存在饱和电流, 因此1D1R结构没有限流功 能，这两方面的因素严重影响了其在高密度集成中 的应用. 鉴于此, $\mathrm{Li}$ 等人 ${ }^{[33]}$ 提出了自限流的双极性 1D1R 结构。与传统的单极性 1D1R相比, 双极性 1D1R采用肖特基二极管的反向电流作为钳制电流, 采用肖特基二极管的正向电流完成擦除过程，该结 构的提出为克服 $1 \mathrm{D} 1 \mathrm{R}$ 结构固有的缺陷 (无限流功能 以及不适用于双极性RRAM) 提供了新思路. 图3(a) 为制备的 $\mathrm{Ti}^{2} / \mathrm{TiO}_{x} / \mathrm{Ti}$ 以及 $\mathrm{Ni} / \mathrm{TiO}_{x} / \mathrm{Ti}$ 器件的 $I-V$ 特性曲 线. 从图中可以发现, $\mathrm{Ti} / \mathrm{TiO}_{x} / \mathrm{Ti}$ 器件在正负电压范围 内具有对称的 $I-V$ 特性, 并且漏电流大, 意味着金属 $\mathrm{Ti}$ 与具有 $\mathrm{n}$ 型半导体特性的 $\mathrm{TiO}_{x}$ 界面形成了欧姆接触. 当采用高功函数的金属 $\mathrm{Ni}$ 作为上电极构成 $\mathrm{Ni} / \mathrm{TiO}_{x} / \mathrm{Ti}$ 器件, 其表现出明显的整流特性. 从图3(a)中可以发 现 $\mathrm{Ni} / \mathrm{TiO}_{x} / \mathrm{Ti}^{\mathrm{g}}$ 件的反向电流随着电压的增大逐渐增 大, 当反向电压达到 $-1 \mathrm{~V}$ 时, 反向电流能够达到 100 $\mu \mathrm{A}$, 如此大的电流可以与双极性的RRAM器件集成 构成 $1 \mathrm{D} 1 \mathrm{R}$ 结构. 图 3(b) 所示为制备的 $\mathrm{Pt} / \mathrm{HfO}_{2} / \mathrm{Cu}$
RRAM存储单元的 $I-V$ 特性曲线. 从图中可以看出, $\mathrm{Pt} / \mathrm{HfO}_{2} / \mathrm{Cu}$ 结构 RRAM存储单元表现出明显的双极 性电阻转变特性. 当反向电压达到一定值时, 器件由 高阻态转变为低阻态, 器件处于低阻态时, 施加正向 电压, 当电压达到一定值时, 器件又由低阻态转变回 高阻态. 图3(c) 所示为 $\mathrm{Pt} / \mathrm{HfO}_{2} / \mathrm{Cu} / \mathrm{Ti} / \mathrm{Ni} / \mathrm{TiO}_{x} / \mathrm{Ti}$ 结构 $1 \mathrm{D} 1 \mathrm{R}$ 存储器件的 $I-V$ 特性曲线. 从图中看出, 该 $1 \mathrm{D} 1 \mathrm{R}$ 结构展现出明显的双极性电阻转变特性. 当 $0 \mathrm{~V} \rightarrow-2.5 \mathrm{~V}$ 的电压施加在Pt电极上, 1D1R 存储器件 的电流在 $-0.5 \mathrm{~V}$ 时快速增加, 意味着器件实现了由高 阻态向低阻态的转变. 当采用 $0 \mathrm{~V} \rightarrow 2.5 \mathrm{~V}$ 的电压, 在 电压值达到 $0.8 \mathrm{~V}$ 时, 器件的电阻突然增大, 说明器 件又转变回了高阻态. 图 3(d) 所示为 $\mathrm{Pt} / \mathrm{HfO}_{2} / \mathrm{Cu} / \mathrm{Ti} /$ $\mathrm{Ni} / \mathrm{TiO}_{x} / \mathrm{Ti}$ 结构 $1 \mathrm{D} 1 \mathrm{R}$ 存储器的可重复性特性. 从图 $3(\mathrm{~d})$ 可以发现, 该双极性的 1D1R 存储器件具有稳定 的可重复性, 在 100 次连续的直流扫描周期下, 器件 依然具有稳定的电阻转变特性. 此外, 这种双极性的 1D1R存储器件在由高阻态向低阻态转变 (SET)过程 过程中能够实现自整流的特性, 意味着该器件结构 在实现SET的过程中不需要施加额外的限制电流，大 大降低了RRAM器件外围电路设计的难度.

基于以上研究结果，1D1R结构是采用二极管来 选择所需操作的存储单元并抑制串扰现象, 选择二 极管是否具有较大的正向电流密度(forward current density)、较高的整流比(F/R ratio)、能否与阻变单元 实现三维集成是基于 $1 \mathrm{D} 1 \mathrm{R}$ 结构交叉阵列实现高集成 密度的关键因素 ${ }^{[25]}$. 因为 $\mathrm{Si}$ 二极管在制备过程中需 要高的制备温度, 这不利于后端工艺的集成, 而基于 

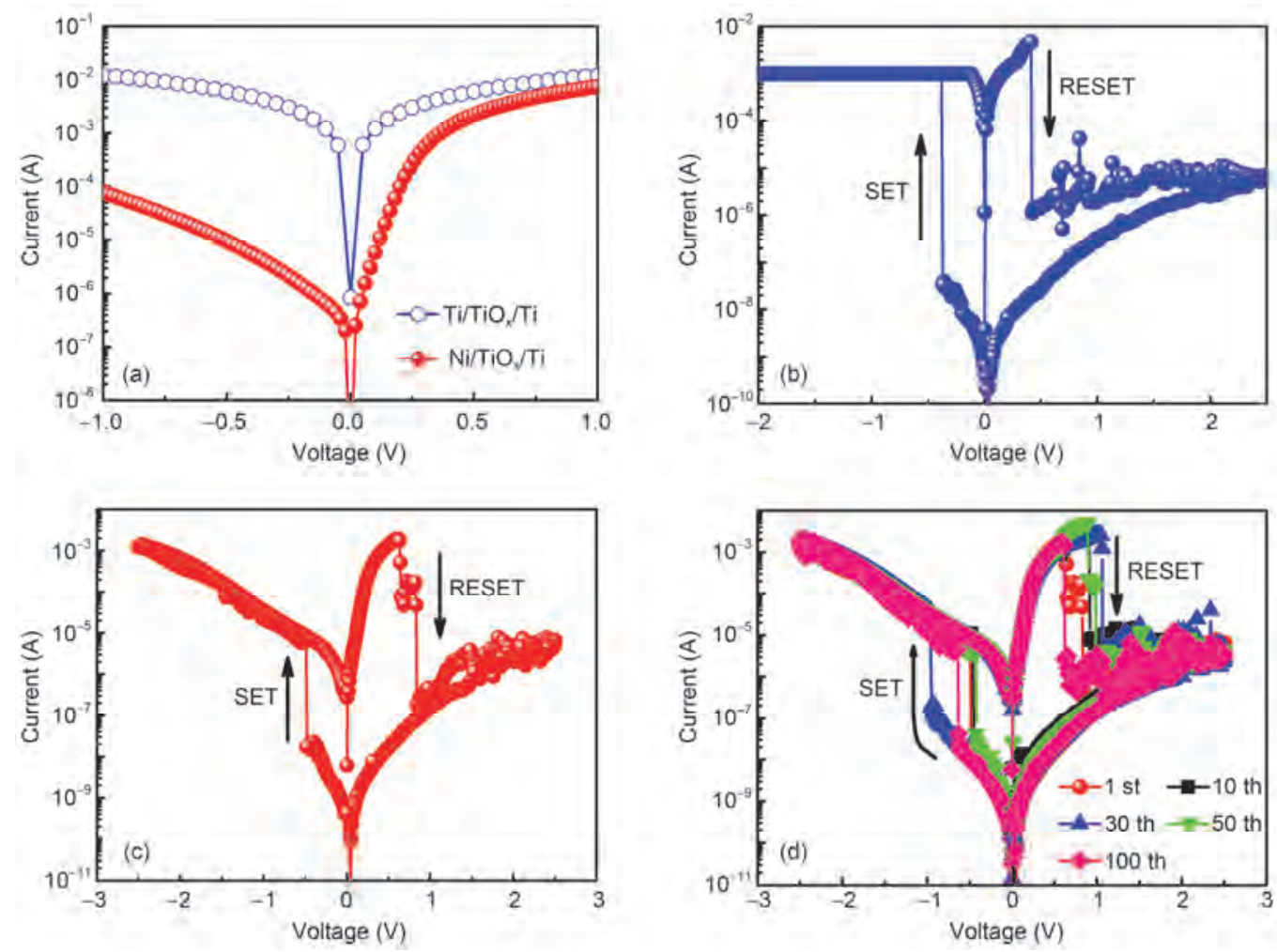

图 3 (网络版彩色)自限流双极性 1D1R结构的电流电压特性. (a) $\mathrm{Ti}^{2} / \mathrm{TiO}_{x} / \mathrm{Ti}$ 和 $\mathrm{Ni}^{\prime} / \mathrm{TiO}_{x} / \mathrm{Ti}$ 器件的I-V特性; (b) $\mathrm{Pt} / \mathrm{HfO}_{2} / \mathrm{Cu}$ RRAM存储单元的双极 性电阻转变特性; (c) $\mathrm{Pt} / \mathrm{HfO}_{2} / \mathrm{Cu} / \mathrm{Ti} / \mathrm{Ni} / \mathrm{TiO}_{x} / \mathrm{Ti}_{1} 1 \mathrm{D} 1 \mathrm{R}$ 存储器件的I-V特性; (d) $\mathrm{Pt} / \mathrm{HfO}_{2} / \mathrm{Cu} / \mathrm{Ti} / \mathrm{Ni}^{2} / \mathrm{TiO}_{x} / \mathrm{Ti} 1 \mathrm{D} 1 \mathrm{R}$ 存储器件的可重复特性 $[33]$

Figure 3 (Color online) $I-V$ characteristics of self-compliance bipolar $1 \mathrm{D} 1 \mathrm{R}$ structure. (a) Typical $I-V$ curves of $\mathrm{Ti} / \mathrm{TiO}_{x} / \mathrm{Ti}$ and $\mathrm{Ni} / \mathrm{TiO} \mathrm{x}_{\mathrm{x}} / \mathrm{Ti}$ devices; (b) bipolar resistive switching characteristics of $\mathrm{Pt} / \mathrm{HfO}_{2} / \mathrm{Cu} \mathrm{RRAM}$ cell; (c) $I-V$ curve of $\mathrm{Pt} / \mathrm{HfO}_{2} / \mathrm{Cu} / \mathrm{Ti} / \mathrm{Ni} / \mathrm{TiO} / \mathrm{Ti} 1 \mathrm{D} 1 \mathrm{R}$ memory device; (d) reproducibility of the bipolar switching characteristics of $\mathrm{Pt} / \mathrm{HfO}_{2} / \mathrm{Cu} / \mathrm{Ti} / \mathrm{Ni} / \mathrm{TiO}_{x} / \mathrm{Ti} 1 \mathrm{D} 1 \mathrm{R}$ structure ${ }^{[33]}$

金属氧化物材料的二极管具有制备温度低、与CMOS 工艺兼容性良好的特点, 因此备受关注. 然而, 目前 基于金属氧化物材料的二极管的电流密度还不是很 高, 当RRAM存储单元的器件面积持续缩小时, 二极 管提供的电流不足以使阻变存储器发生电阻转变, 为了能够提供足够大的驱动电流, 通常则需将二极 管单元的尺寸做的比RRAM单元的尺寸大, 导致存 储单元的面积大于 $4 \mathrm{~F}^{2}$. 因此, 基于 $1 \mathrm{D} 1 \mathrm{R}$ 结构的集成 方案在很大程度上依赖于整流二极管的性能, 二极管 的性能指标成为研究过程中最棘手的问题, 能否研究 出正向电流密度大、整流比高、制备温度低、易于三 维集成的二极管是1D1R集成实现高密度的关键.

\section{1S1R结构}

$1 \mathrm{~S} 1 \mathrm{R}$ 结构是由一个电学特性对称的非线性电阻 与阻变存储器件串联构成的, 其中阻变存储单元具 有稳定的双极性电阻转变特性. Huang等人 ${ }^{[34]}$ 将一个 电学特性对称的非线性电阻 $\mathrm{Ni} / \mathrm{TiO}_{2} / \mathrm{Ni}$ 作为选通管,
与阻变存储器 $\mathrm{Ni} / \mathrm{HfO}_{2} / \mathrm{Pt}$ 串联, 如图4所示, 当 $\mathrm{V}_{\mathrm{SET}}$ 大 于选通管的开启电压 $\mathrm{V}_{\mathrm{th}}$ 时, 存储器件由高阻态转变 为低阻态, 当电流回扫, 电压小于 $\mathrm{V}_{\mathrm{th}}$ 时, 直接表现为 选通管的电学性质.

目前，1S1R结构存在的主要问题之一是非线性 系数低, 在其集成时, 影响了阵列的大小 ${ }^{[34]}$. 因此寻 找非线性系数大的对称性非线性电阻是发展 $1 S 1 R$ 结 构的主要方向. Luo等人 ${ }^{[35]}$ 将外加引人隧穿层的一个 电学特性对称的非线性电阻 $\mathrm{Cu} / \mathrm{doped} \mathrm{Cu}-\mathrm{HfO}_{2} / \mathrm{Pt}$ 作 为选通管, 与阻变存储器串联, 构成 $1 \mathrm{~S} 1 \mathrm{R}$ 结构. 该选 通管最大的电流密度大于 $1 \mathrm{MA} / \mathrm{cm}^{2}$, 非线性系数高 达 $10^{7}$, 耐久性可以持续 $10^{10}$ 个循环, 充足的读取余度 可保证 $10^{5} \mathrm{~Gb}$ 的阵列尺寸, $\mathrm{pA}$ 量级的漏电流可以有 效抑制无源交叉阵列中的寄生电流, 并且降低功耗, Lee小组 ${ }^{[36]}$ 报道了器件结构为 $\mathrm{Pt} / \mathrm{TaO}_{x} / \mathrm{TiO}_{2} / \mathrm{TaO}_{x} / \mathrm{Pt}$ 的 非线性电阻, 其最大的电流密度高于 $10^{7} \mathrm{~A} / \mathrm{cm}^{2}$, 并 且非线性系数达 $10^{4}$, 如图 5 所示.

2017年, Rivu等人 ${ }^{[37]}$ 报道的挥发性阈值开关选择 


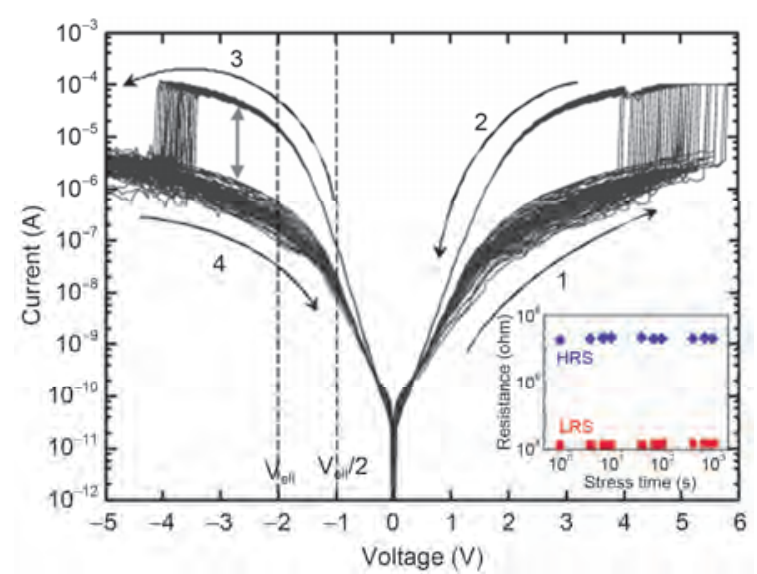

图 4 (网络版彩色) $\mathrm{Ni} / \mathrm{TiO}_{2} / \mathrm{Ni} / \mathrm{HfO}_{2} / \mathrm{Pt}$ 集成 $1 \mathrm{~S} 1 \mathrm{R}$ 的电学特性曲线 ${ }^{[34]}$ Figure 4 (Color online) $I-V$ characteristics of $\mathrm{Ni} / \mathrm{TiO}_{2} / \mathrm{Ni} / \mathrm{HfO}_{2} / \mathrm{Pt}$ 1S1R structure ${ }^{[34]}$

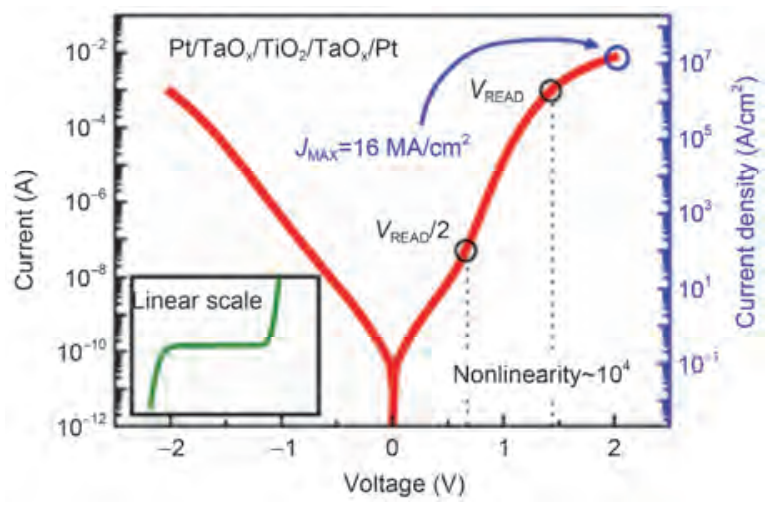

图 5 (网络版彩色) $\mathrm{Pt} / \mathrm{TaO}_{X} / \mathrm{TiO}_{2} / \mathrm{TaO}_{x} / \mathrm{Pt}$ 器件的电学特性曲线 ${ }^{[36]}$

Figure 5 (Color online) Typical $I-V$ characteristic of $\mathrm{Pt} / \mathrm{TaO}_{x} / \mathrm{TiO}_{2} /$ $\mathrm{TaO}_{x} / \mathrm{Pt}$ structure ${ }^{[36]}$

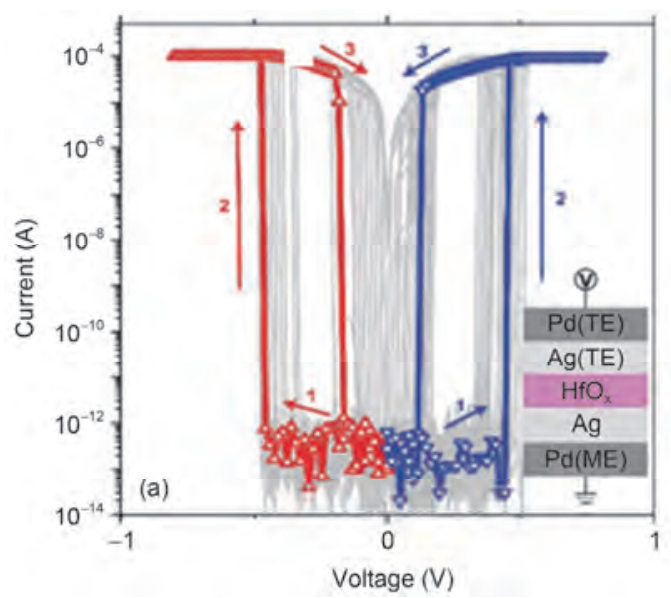

器 $\mathrm{Pd} / \mathrm{Ag} / \mathrm{HfO}_{x} / \mathrm{Ag} / \mathrm{Pd}$ 的非线性系数达到了 $10^{10}$, 耐久 性可以持续 $10^{8}$ 个循环, 开启斜率小于 $1 \mathrm{mv} / \mathrm{dec}$, 是至 今报道的斜率最大值，开/关速度小于 $75 / 250 \mathrm{~ns}$ ，关 断漏电流为 $10^{-14} \mathrm{~A}$, 该电流是迄今为止报道的最小 值，如图6(a)所示. 将该结构作为选通管，与双极性 阻变存储器 $\mathrm{Pd} / \mathrm{TaO}_{x} / \mathrm{Ta}_{2} \mathrm{O}_{5} / \mathrm{Pd}$ (图6(b) 是该阻变存储器 的电流电压特性曲线)串联构成 $1 \mathrm{~S} 1 \mathrm{R}$ 结构, 电压电流 特性如图7所示, 与单独的阻变存储器相比, 很大程 度地抑制了交叉阵列结构中的漏电流.

Qing等人 ${ }^{[38]}$ 制备出的基于梯形能带结构, 在于得 到一致性和非线性系数高的非线性电阻 $\mathrm{Ru} / \mathrm{TaO}_{x} / \mathrm{W}$, 其电流密度大于 $1 \mathrm{MA} / \mathrm{cm}^{2}$, 非线性系数大于 $5 \times 10^{4}$, 耐久性可以持续 $10^{10}$ 个循环, 关断漏电流约为 $10 \mathrm{pA}$. 将其作为选通管与双极性阻变存储器 $\mathrm{Cu} / \mathrm{HfO}_{2} / \mathrm{Pt}$ 串 联构成 $1 \mathrm{~S} 1 \mathrm{R}$ 结构, 取合适的读取电压, 低阻态下的 整流比可达 $10^{4}, 85^{\circ} \mathrm{C}$ 下的数据保持特性大于 $10^{4} \mathrm{~s}$, 在高密度无源交叉阵列中有很大的应用潜力. 最近 中国科学院微电子研究所刘明院士团队 ${ }^{[39]}$ 提出通过 石墨烯缺陷工程控制活性电极离子向阻变功能层中 注人的路径尺寸和数量, 集中化/离散化阳离子基阻 变器件中导电通路的分布来调控其稳定性, 不仅获 得了高驱动电流(双向 $500 \mu \mathrm{A}$ ) 的易失性國值开关选 择器(threshold selector), 结构为 Ag/Defect Graphene/ $\mathrm{SiO}_{2} / \mathrm{Pt}$, 而且获得了低操作电流 $(1 \mu \mathrm{A})$ 、高保持特性、 低功耗的非易失性阻变存储器, 这为阻变存储器的 $1 \mathrm{~S} 1 \mathrm{R}$ 结构三维高密度集成奠定了基础. 此工作是该

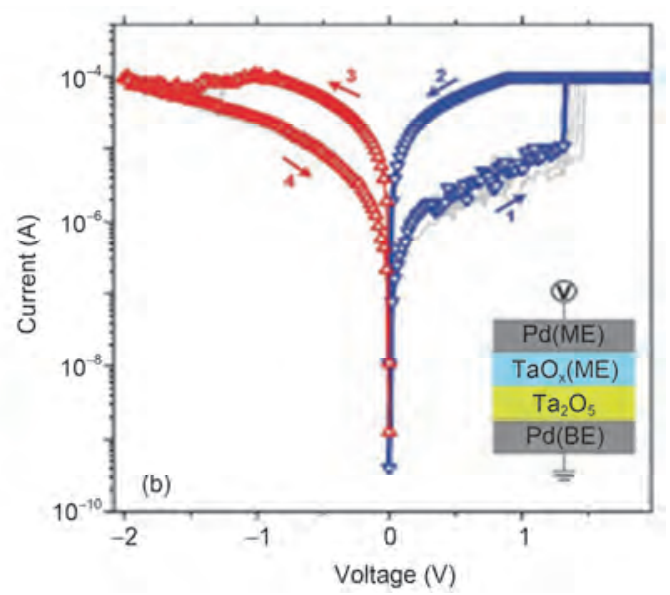

图 6 (网络版彩色)非线性电阻与阻变器件的电流电压特性. (a) 非线性电阻 $\mathrm{Pd} / \mathrm{Ag} / \mathrm{HfO}_{x} / \mathrm{Ag} / \mathrm{Pd}$ 结构的电流电压特性曲线; (b) 阻变存储器 $\mathrm{Pd} / \mathrm{TaO}_{x} / \mathrm{Ta}_{2} \mathrm{O}_{5} / \mathrm{Pd}$ 电流电压特性曲线 ${ }^{[37]}$

Figure 6 (Color online) $I-V$ characteristics of the selector and RRAM devices. (a) Repeatable bidirectional threshold switching of the $\mathrm{Pd} / \mathrm{Ag} / \mathrm{HfO}_{x} / \mathrm{Ag} / \mathrm{Pd}$ selector; (b) $I-V$ curves of the $\mathrm{Pd} / \mathrm{Ta}_{2} \mathrm{O}_{5} / \mathrm{TaO}_{x} / \mathrm{Pd} \mathrm{RRAM}$ cell ${ }^{[37]}$ 


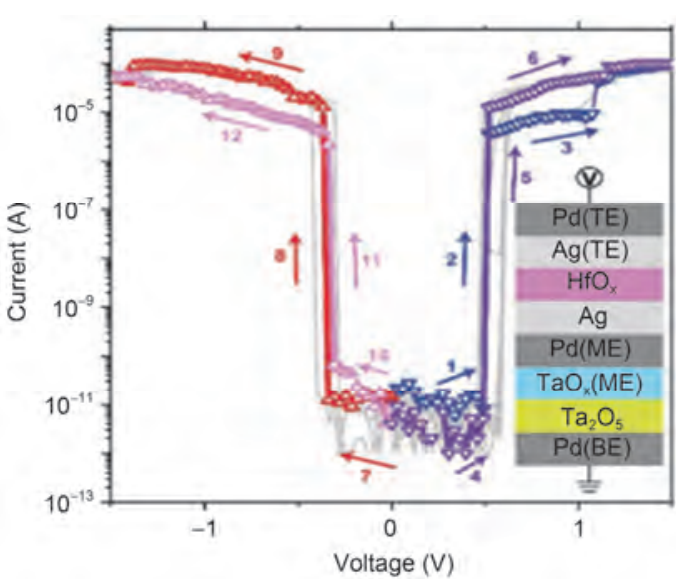

图 7 (网络版彩色) $1 \mathrm{~S} 1 \mathrm{R}$ 结构 $\mathrm{Pd} / \mathrm{Ag} / \mathrm{HfO}_{x} / \mathrm{Ag} / \mathrm{Pd} / \mathrm{TaO}_{x} / \mathrm{Ta}_{2} \mathrm{O}_{5} / \mathrm{Pd}$ 电流 电压特性曲线 ${ }^{[37]}$

Figure 7 (Color online) $I-V$ chatacteristics of $\mathrm{Pd} / \mathrm{Ag} / \mathrm{HfO}_{x} / \mathrm{Ag} / \mathrm{Pd} /$ $\mathrm{TaO}_{x} / \mathrm{Ta}_{2} \mathrm{O}_{5} / \mathrm{Pd}$ 1S1R structure ${ }^{[37]}$

领域首次在相同结构阻变器件中实现电流-保持特性 的双向调控, 这种通用的基于二维材料阻挡概念的 离子迁移调控方法, 也能够移植应用到离子电池、离 子传感等研究领域.

表2给出了部分目前研究报道的可用于 $1 \mathrm{~S} 1 \mathrm{R}$ 集 成的非线性电阻选择器的性能参数. 与 $1 \mathrm{D} 1 \mathrm{R}$ 结构相 比, $1 \mathrm{~S} 1 \mathrm{R}$ 结构中选通器件具有双极对称性, 因而对 RRAM的器件极性没有限制, 而且这种结构对 RRAM的编程和读取操作能够在同向进行, 在减少 漏电和降低功耗方面要明显优于1D1R结构.

\section{5 互补电阻开关结构(back to back)}

互补电阻开关(complementary resistive switches, CRS)也称背靠背结构, 如图8所示, 是由两个双极转 变的阻变存储元件反向串联构成的, 该概念是Linn
等人 ${ }^{[44]}$ 在 2010 年提出的. CRS 结构具有特殊的读取性 质, 结构最初状态为 $\mathrm{OFF}$ 状态, 此时存储单元 $\mathrm{A} 、 \mathrm{~B}$ 都 处于高阻态; 当CRS的状态处于 “ 0 ”时, A为高阻态, B 为低阻态; 当 $C R S$ 的状态处于 “ 1 ”时, $A$ 为低阻态, $B$ 为 高阻态; 当CRS的处于“ON”状态时, A、B都处于低阻 态, 这种状态仅在读操作时才会发生. 一经提出, 这 一概念便受到了科研界的广泛关注. 2011年, Lee小 组 ${ }^{[45]}$ 提出了基于背靠背概念的 $\mathrm{Pt} / \mathrm{Ta}_{2} \mathrm{O}_{5-x} / \mathrm{TaO}_{2-x} / \mathrm{Pt} /$ $\mathrm{TaO}_{2-x} / \mathrm{Ta}_{2} \mathrm{O}_{5-x} / \mathrm{Pt}$ 结构, 该结构器件由低阻态向高祖 态转变 (RESET) 的电流仅为 $50 \mu \mathrm{A}$, 并且能够完成 $10^{12}$ 周期的循环操作.

Breuer等人 ${ }^{[46]}$ 实现了基于 $\mathrm{TaO}_{x}$ 垂直堆叠的微米 和纳米级 CRS 结构, 该结构具有低开关操作电流 ( 200 $\mu \mathrm{A})$, 且耐久性可以持续 $1.25 \times 10^{6}$ 个周期. 此 外, CRS时序逻辑运算首次实现, 包括微米和纳米级 CRS结构的逻辑与运算, 以及纳米级CRS结构的逻辑 蕴含运算, 为基于 $\mathrm{CRS}$ 的逻辑内存操作提供了概念 验证测量和模拟. Shi等人 ${ }^{[47]}$ 最近提出了一种基于石 墨烯氧化物 (graphene oxide, GO)材料的CRS存储器 件, 结构为 $\mathrm{Al} / \mathrm{GO} / \mathrm{ITO}$. 研究表明该结构的阻变过程 在很大程度上依赖于器件开启和存储过程中限制电 流的大小, 过大的限制电流使器件难达到 CRS结构 存储，在该结构中过程限制电流值约为 $200 \mu \mathrm{A}$. 同样 在存储过程中发现如果电流不受限, 就会呈现典型的 CRS结构 $I-V$ 特性, 而当限制电流小于 $500 \mu \mathrm{A}$ 时, 器件 就会呈现双极性阻变存储(bipolar resistive switching, $\mathrm{BRS}$ )特性, 所以该器件结构表现出BRS和 CRS特性 的共存. 高度一致的正反阻变电压使得 CRS结构的 “0”和“1”状态能够清晰地区分，而GO层中的氧空位 对此起着非常重要的作用，同样也注意到该基于石

\section{表 2 部分报道的可用于 1S1R集成的非线性电阻选择器}

Table 2 Comparison of nonlinear resistor selectors for 1S1R integration

\begin{tabular}{|c|c|c|c|c|}
\hline 器件结构 & 最大电流密度 $\left(\mathrm{A} / \mathrm{cm}^{2}\right)$ & 非线性系数 & 可重复性 & 高阻态漏电流 $(\mathrm{A})$ \\
\hline $\mathrm{Cu} /$ doped $\mathrm{Cu}-\mathrm{HfO}_{2} / \mathrm{Pt}^{[35]}$ & $10^{6}$ & $10^{7}$ & $10^{10}$ & $10^{-12}$ \\
\hline $\mathrm{Pd} / \mathrm{Ag} / \mathrm{HfO}_{x} / \mathrm{Ag} / \mathrm{Pd}^{[36]}$ & - & $10^{10}$ & $10^{8}$ & $10^{-14}$ \\
\hline $\mathrm{Pt} / \mathrm{TaO}_{x} / \mathrm{TiO}_{2} / \mathrm{TaO}_{x} / \mathrm{Pt}^{[37]}$ & $10^{7}$ & $10^{4}$ & $10^{10}$ & $10^{-7}$ \\
\hline $\mathrm{Ru} / \mathrm{TaO} x / \mathrm{W}^{[38]}$ & $10^{6}$ & $>5 \times 10^{4}$ & $10^{10}$ & $10^{-11}$ \\
\hline $\mathrm{Ag} /$ Defect Graphene/SiO2/Pt ${ }^{[39]}$ & - & $5 \times 10^{8}$ & $>10^{6}$ & $10^{-13}$ \\
\hline $\mathrm{BE} /$ doped-chalcogenide/ $\mathrm{SiO}_{2} / \mathrm{TE}^{[40]}$ & $1.6 \times 10^{6}$ & $>10^{7}$ & $>10^{9}$ & $10^{-11}$ \\
\hline $\mathrm{BE} / \mathrm{SLT} / \mathrm{TE}^{[41]}$ & $5 \times 10^{6}$ & $10^{10}$ & $>10^{8}$ & $10^{-11}$ \\
\hline $\mathrm{BE} / \mathrm{AsTeGeSiN} / \mathrm{TE}^{[42]}$ & $1.1 \times 10^{7}$ & $10^{4}$ & $10^{8}$ & $10^{-6}$ \\
\hline $\mathrm{TiN} / \mathrm{NbO}_{2} / \mathrm{Pt}^{[43]}$ & $8 \times 10^{7}$ & $10^{3}$ & $10^{3}$ & $10^{-6}$ \\
\hline
\end{tabular}


(a)

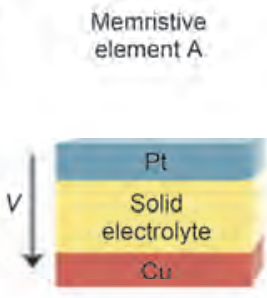

(c)

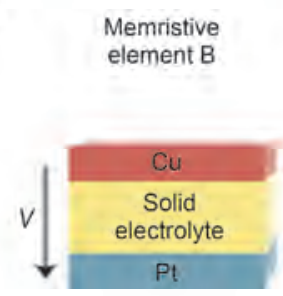

(e)

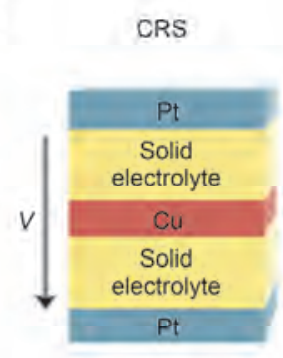

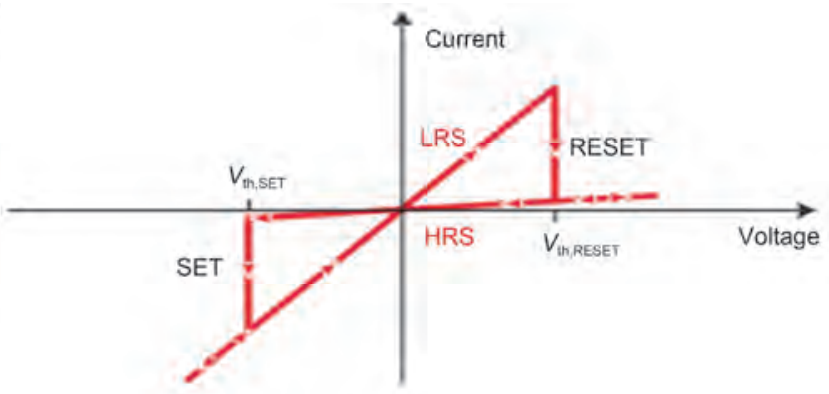

(d)

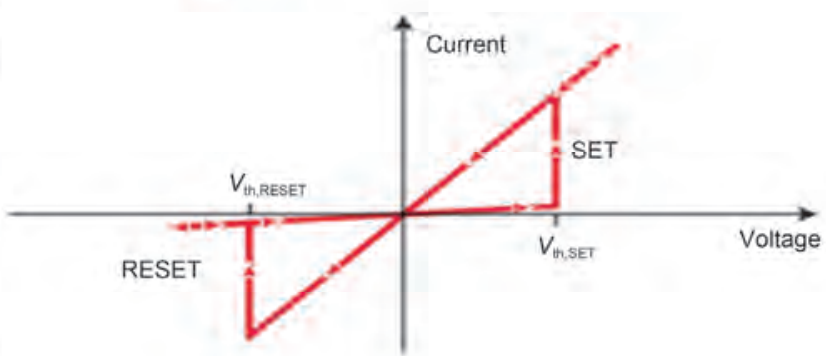

(f)

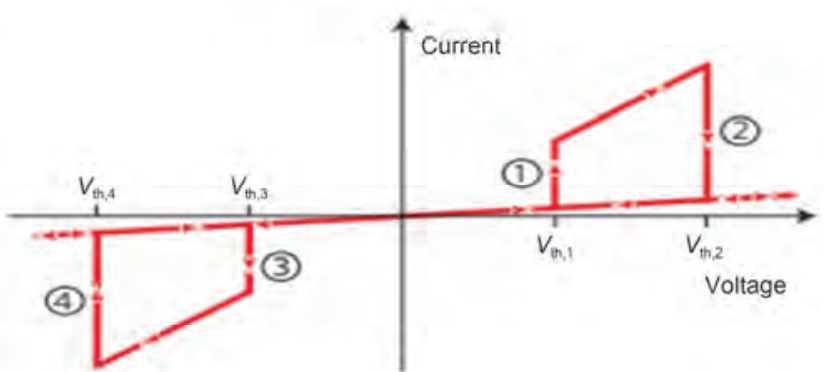

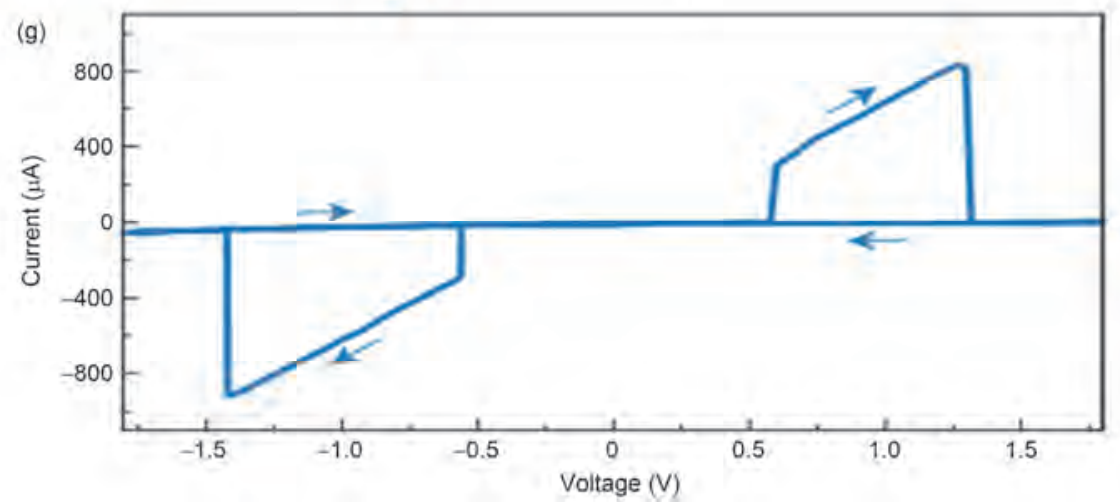

图 8 (网络版彩色) CRS结构的特性. (a) 双极性存储元件A; (b) 存储元件A的 $I-V$ 特性; (c) 双极性存储元件B; (d) 存储元件B的 $I-V$ 特性; (e) 存 储元件A与B串联成CRS结构; (f) CRS结构的 $I-V$ 特性; (g) Pt/SiO$/ / \mathrm{GeSe} / \mathrm{Cu} / \mathrm{GeSe} / \mathrm{SiO}_{2} / \mathrm{Pt}$ 结构器件的 $I-V$ 特性 ${ }^{[44]}$

Figure 8 (Color online) Characteristics of CRS structure. (a) Bipolar memory element A; (b) $I-V$ characteristics of memory element A; (c) bipolar memory element $\mathrm{B}$; (d) $I$ - $V$ characteristics of memory cell B; (e) CRS resulting from the combination of element A and B; (f) $I$ - $V$ characteristics of CRS structure; (g) $I$ - $V$ characteristics of $\mathrm{Pt} / \mathrm{SiO}_{2} / \mathrm{GeSe} / \mathrm{Cu} / \mathrm{GeSe} / \mathrm{SiO}_{2} / \mathrm{Pt}$ structure device ${ }^{[44]}$

墨烯氧化物的阻变存储器在高阻态时的漏电流比较 大, 不仅会增加功耗, 而且限制交叉阵列的密度, 因 此, 在未来的工作中需要进一步的优化器件结构.

虽然CSR结构可以抑制无源交叉阵列中串扰问 题, 但是由于高低阻态的开关比较低, 存储窗口较
小，限制了阵列的大小. 此外由于 CRS结构存储“ “0” 和 “ 1 ”时, 电阻状态都是高阻, 判断其状态之前需要 加电压预处理, 多次的预处理操作很容易造成存储 单元的损伤，会减小其使用寿命.

2011 年三星公司 ${ }^{[48]}$ 提出的 $1 \mathrm{TS} 1 \mathrm{R}$ 结构, 其 $I-V$ 特 
性与上文所提的背靠背结构的 $I-V$ 特性相似, 但两者 不是同一种结构, 其原理以及阻变过程皆不同. 1TS1R 是由一个具有阈值开启 (threshold switching, $\mathrm{TS}$ )特性的开关器件和一个双极转变的阻变存储元件 串联构成的. TS是一种易失性存储器件, 如图6所示 为具有 $\mathrm{Pd} / \mathrm{Ag} / \mathrm{HfO}_{x} / \mathrm{Ag} / \mathrm{Pd}$ 结构的阈值开关选择器 ${ }^{[37]}$, 它可以通过控制施加电压的极性和大小呈现两种不 同的易失性电阻状态, 与双极性阻变存储单元串联 后, 当施加读取电压 $V_{\mathrm{READ}}$ 时, 可以读取 $1 \mathrm{R}$ 存储单元 的状态, 即可以确定 $1 \mathrm{R}$ 单元的 ON/OFF状态. 另一方 面, 当施加 $V_{\mathrm{READ} / 2}$ 读取电压时, $1 \mathrm{TS}$ 始终处于关闭状 态, 这会阻止电流流过 $1 \mathrm{TS} 1 \mathrm{R}$ 器件, 此操作可以解决 漏电通路的问题, 为了 $1 \mathrm{TS} 1 \mathrm{R}$ 结构的正常运行, 需要 满足以下条件: $V_{\mathrm{th}}<\left|V_{\mathrm{RESET}}\right|$ 和 $V_{\mathrm{th}}<V_{\mathrm{SET}}$. 该结构的提出 不仅能有效解决漏电通路问题, 而且其自身也在高 密度低功耗非易失性交叉阵列存储的方向上有着重 要的应用前景.

\section{6 具有自整流特性的1R结构}

自整流器件是抑制阻变存储器交叉阵列结构中 串扰现象的有效手段之一. 所谓自整流, 即阻变存储 单元在具有阻变特性的同时又具有整流特性, 使其 在不需要额外选通管的前提下, 实现自我选择功 能, 可以大大减小器件结构的复杂性. 因此该结构器 件近年来受到了广泛的关注, 并且被大量的研究和 应用。

Tran等人 ${ }^{[49]}$ 报道了具有自整流特性的单极性 $\mathrm{NiSi} / \mathrm{HfO}_{x} / \mathrm{TiN}$ 器件, 其低阻态下的整流比大于 $10^{3}$ $(1 \mathrm{~V})$, 该存储器件的开关比约为 $10^{2}, 125^{\circ} \mathrm{C}$ 下的数据 保持特性达 $10^{5} \mathrm{~s}$, 该结构器件能够实现 $2^{6} \times 2^{6}$ 的交叉 存储阵列. 该小组接着又报道了具有自整流特性的 双极性结构 $\mathrm{Ni} / \mathrm{AlO}_{y} / \mathrm{n}^{+}-\mathrm{Si}$ 器件 ${ }^{[50]}$, 其低阻态下的整流 比大于 $700(0.2 \mathrm{~V})$, 这种存储器件的开关比大约为 $10^{3}, 100^{\circ} \mathrm{C}$ 下的数据保持特性达 $10^{4} \mathrm{~s}$, 能够实现 $2^{5} \times 2^{5}$ 的交叉存储阵列. $\mathrm{Li}$ 等人 ${ }^{[51]}$ 在 2014 年报道了基于 $\mathrm{HfO}_{2}$ 和 $\mathrm{SiO}_{2}$ 薄膜材料的自整流器件单元, 分别采用 $\mathrm{Ni}$ 和 $\mathrm{n}^{+}-\mathrm{Si}$ 作为上下电极制备了具有 $\mathrm{Ni} / \mathrm{HfO}_{2} / \mathrm{n}^{+}-\mathrm{Si}$ 和 $\mathrm{Ni} / \mathrm{SiO}_{2} / \mathrm{n}^{+}-\mathrm{Si}$ 结构的阻变存储器件单元, 发现 $\mathrm{Ni} /$ $\mathrm{HfO}_{2} / \mathrm{n}^{+}-\mathrm{Si}$ 和 $\mathrm{Ni} / \mathrm{SiO}_{2} / \mathrm{n}^{+}-\mathrm{Si}$ 器件在低阻态时都具有明 显的整流特性, 这种低阻态的整流特性能够有效抑 制交叉阵列中的串扰现象.

中国科学院微电子研究所 ${ }^{[52]}$ 报道了以非晶硅作
为插层材料的自整流器件, 利用阻变材料层与非晶 硅层的肖特基接触实现自整流特性. 通过对比研究 可以判断 $\mathrm{Cu} / \mathrm{a}-\mathrm{Si} / \mathrm{WO}_{3} / \mathrm{Pt}$ 器件的整流特性来自于 $\mathrm{a}-\mathrm{Si} / \mathrm{WO}_{3}$ 的界面. 由于在初始开启过程中, $\mathrm{WO}_{3}$ 层内 部形成了由氧空位构成的导电通道, 自整流现象的 本质则是 $\mathrm{WO}_{3}$ 中氧空位通道与 $\mathrm{a}-\mathrm{Si}$ 之间形成的肖特 基接触. 所制备的自整流 $\mathrm{Cu} / \mathrm{a}-\mathrm{Si} / \mathrm{WO}_{3} / \mathrm{Pt}$ 器件具有非 常优越的重复性和一致性, 在经过连续 1000 次的 $I-V$ 曲线扫描后，扫描得到的曲线和第一次的曲线基本 重合. 此外, 该结构器件的Reset电流很小, 可以达到 小于微安的量级, 可适合于低功耗场合的应用. 同 时, 中国科学院微电子研究所 ${ }^{[53]}$ 利用具有自整流特 性的 $\mathrm{Pt} / \mathrm{ZrO}_{2} / \mathrm{n}^{+}-\mathrm{Si}$ 结构存储器制备了一次编程存储器 (WORM), 该器件编程到低阻态时能够永久保持, 并 且整流比大于 $10^{4}$, 开关比约为 $10^{6}$, 而且在编程前后 电阻分布集中, 室温下的数据保持特性为 $10^{5} \mathrm{~s}$ (如图 9 所示).

表3给出了部分报道的有自整流特性的RRAM器 件的性能参数. 具有自整流特性的阻变存储器结构 简单、性能稳定、与CMOS工艺兼容, 并且由于没有 选择器件引人的干扰, 克服了操作电压增大、重复性 变差等缺点. 这些优势使得自整流阻变存储器在高 密度无源交叉阵列结构的应用中具有较强的竞争力.

\section{7 结论与展望}

基于无源交叉阵列的RRAM具有高密度、易于三 维集成的优势, 因此最有可能在未来的高密度 RRAM产品中有广阔的应用前景，在无源交叉阵列 结构中, 由于泄漏电流的存在, 通常需要与RRAM单 元串联一个选通管来抑制交叉串扰引起的误读现象, 目前采取的解决方法主要包括：(1) 串联一个具有整 流特性的二极管，构成1D1R结构; (2) 串联一个非线 性电阻, 构成1S1R结构; (3) 串联一个互补电阻开关 器件, 构成1CRS1R结构; (4) 自整流RRAM器件. 对 基于RRAM器件的高密度无源交叉阵列来说, 虽然 目前已经取得了一些成功, 然而RRAM无源交叉阵 列的高密度集成应用依然面临许多挑战, 更多重要 工作仍在进行中, 更多问题仍需解决. 对于 $1 \mathrm{D} 1 \mathrm{R}$ 结 构, 能否找到具有高的电流密度、大的整流比和低温 工艺的整流二极管，是决定 $1 \mathrm{D} 1 \mathrm{R}$ 结构能否集成应用 的关键. 对于 $1 \mathrm{~S} 1 \mathrm{R}$ 和 $1 \mathrm{CRS} 1 \mathrm{R}$ 结构集成中的关键问 题, 如材料的选取、非线性系数的优化、开关比的提 

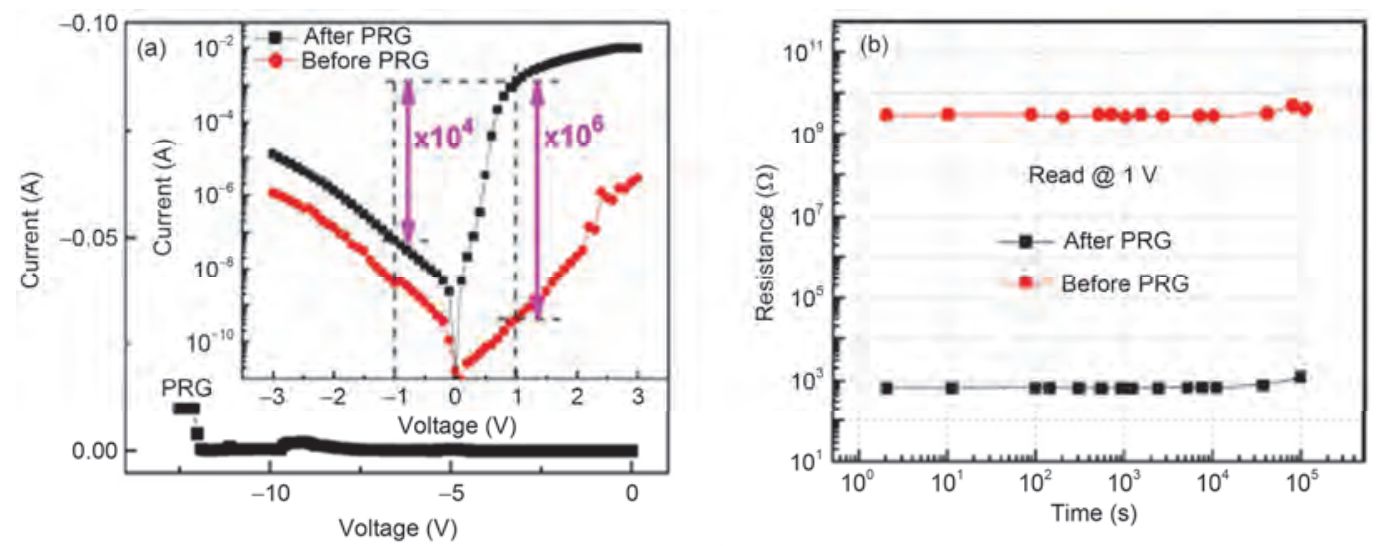

图 9 (网络版彩色)WORM器件的电流电压特性. (a) WORM器件的编程过程(PRG)及其电流-电压曲线; (b) 室温下数据保持特性统计分布图 ${ }^{[33]}$ Figure 9 (Color online) $I-V$ characteristics of the WORM device. (a) Programming process (PRG) for the fresh device. Inset is the typical $I$ - $V$ curves of the WORM memory; (b) retention characteristics of the WORM memory at room temperature ${ }^{[53]}$

\section{表 3 部分报道的具有自整流效应的RRAM}

Table 3 Comparison of RRAM with self-rectifying effect

\begin{tabular}{|c|c|c|c|c|}
\hline 器件结构 & 高低阻态比 & 自整流比 & 可重复性 & 保持特性 \\
\hline $\mathrm{NiSi} / \mathrm{HfO}_{x} / \mathrm{TiN}^{[49]}$ & $10^{2}$ & $10^{3} @ 1 \mathrm{~V}$ & - & $10^{5} \mathrm{~s}$ \\
\hline $\mathrm{Ni} / \mathrm{AlO}_{y} / \mathrm{n}^{+}-\mathrm{Si}^{[50]}$ & $10^{3}$ & $700 @ 0.2 \mathrm{~V}$ & - & $10^{4} \mathrm{~s}$ \\
\hline $\mathrm{Pt} / \mathrm{ZrO}_{2} / \mathrm{n}^{+}-\mathrm{Si}^{[53]}$ & $10^{6}$ & $10^{4}$ & - & $10^{5} \mathrm{~s}$ \\
\hline $\mathrm{Ni} / \mathrm{HfO}_{x} / \mathrm{n}^{+}-\mathrm{Si}^{[54]}$ & - & $10^{3} @ 0.5 \mathrm{~V}$ & $>10^{5}$ & $10^{5} \mathrm{~s}$ \\
\hline $\mathrm{TiW} / \mathrm{Ge}_{2} \mathrm{Sb}_{2} \mathrm{Te}_{5} / \mathrm{W}^{[55]}$ & - & - & $>10^{3}$ & $5000 \mathrm{~s}$ \\
\hline $\mathrm{Au} / \mathrm{ZrO}_{2}: \mathrm{Au}-$ nanocystal $/ \mathrm{n}^{+}-\mathrm{Si}^{[56]}$ & - & $700 @ \pm 0.5 \mathrm{~V}$ & 100 & - \\
\hline $\mathrm{Ag} / \mathrm{RbAg}_{4} \mathrm{I}_{5} / \mathrm{n}^{+}-\mathrm{Si}^{[57]}$ & $10^{3} @ 0.2 \mathrm{~V}$ & - & $10^{3}$ & - \\
\hline $\mathrm{Cu} / \mathrm{a}-\mathrm{Si} / \mathrm{WO}_{3} / \mathrm{Pt}^{[52]}$ & $10^{2} @ 0.5 \mathrm{~V}$ & $10^{2} @ \pm 0.75 \mathrm{~V}$ & & \\
\hline $\mathrm{Si} / \mathrm{a}-\mathrm{Si}$ core/Ag nanowires ${ }^{[58]}$ & $10^{4}$ & $10^{6} @ 1 \mathrm{~V}$ & $10^{4}$ & $>2$ 周 \\
\hline $\mathrm{Ag} / \mathrm{a}-\mathrm{Si} / \mathrm{p}-\mathrm{Si}\left(\right.$ crystalline $^{[59]}$ & $10^{3}$ & - & $10^{6}$ & $>3$ 月 \\
\hline Ag nanowires/a-Si/poly-Si ${ }^{[60]}$ & $>10^{6} @ 0.5 \mathrm{~V}$ & $>10^{6} @ \pm 0.5 \mathrm{~V}$ & $>10^{8}$ & $>4$ 年 \\
\hline
\end{tabular}

高等还缺乏系统的研究, 此外, 理论上还缺乏相应模 型的指导. 因此, 寻找性能、可应用性较好的材料, 开展理论机制研究对 $1 \mathrm{~S} 1 \mathrm{R}$ 和 1CRS1R结构的集成应 用具有十分重要的意义. 相比于其他结构集成方案, 具有自整流特性的 $1 R$ 结构集成工艺更加简单、成本 更加低廉, 并且避免了串联晶体管、二极管等选择器 引人的干扰. 这些优势使得自整流RRAM器件在高 密度三维集成应用中具有较强竞争力, 非常有希望 在RRAM高密度存储中最终得到应用. 整流比不够
高、电流密度小是目前自整流RRAM器件存在的主要 问题. 因此, 进一步深人研究阐明自整流RRAM器件 的内在物理机制, 研制出整流比大、电流密度高、均 匀性、稳定性和可靠性俱佳的高性能自整流RRAM器 件是实现自整流RRAM高密读存储应用的关键.

可以预计，随着技术的不断进步、半导体技术的 高速发展、研究工作的不断深人, 阻变存储器无源阵 列将取得巨大突破，有望在未来高密度存储技术中 得到广泛的应用并取得巨大的商业价值。

\section{参考文献}

1 Kim K. From the future Si technology perspective: Challenges and opportunities. In: International Electron Devices Meeting Technical Digest, 2010. 111-119 
2 Meijer G I. Who wins the nonvolatile memory race? Science, 2008, 319: 1625-1626

3 Tehrani S. Status and outlook of MRAM memory technology. International Electron Devices Meeting Technical Digest, 2006. 585-588

4 Zhu J G, Zheng Y F, Prinz G A. Ultrahigh density vertical magnetoresistive random access memory. J Appl Phys, 2000, 87: 6668-6673

5 Lai S, Lowrey T. OUM-a $180 \mathrm{~nm}$ nonvolatile memory cell element technology for stand alone and embedded applications. In: International Electron Devices Meeting Technical Digest, 2001. 803-806

6 Guan W H, Long S B, Liu Q, et al. Nonpolar nonvolatile resistive switching in Cu doped $\mathrm{ZrO}_{2}$. IEEE Electr Device L, 2008, 29: 434-437

7 Simpson R E, Krbal M, Fons P, et al. Toward the ultimate limit of phase change in $\mathrm{Ge}_{2} \mathrm{Sb}_{2} \mathrm{Te}_{2}$. Nano Lett, 2010, 10: 414-419

8 Scott J C. Is there an immortal memory? Science, 2004, 304: 62-63

9 Lee M-J, Park Y, Kang B S, et al. 2-stack 1D-1R cross-point structure with oxide diodes as switch elements for high density resistance RAM applications. In: International Electron Devices Meeting Technical Digest, 2007. 771-774

10 Chen A, Haddad S, Wu Y C, et al. Non-volatile resistive switching for advanced memory applications. In: International Electron Devices Meeting Technical Digest, 2005. 746-749

11 Zhang W W, Pan W, Ulrich B D, et al. Novel colossal magnetoresistive thin film nonvolatile resistance random access memory (RRAM). In: International Electron Devices Meeting Technical Digest, 2002. 193-196

12 Wang C H, Tsai Y H, Lin K C, et al. Three-dimensional $4 \mathrm{~F}^{2}$ ReRAM cell with CMOS logic compatible process. In: International Electron Devices Meeting Technical Digest, 2010. 664-667

13 Servalli G. A $45 \mathrm{~nm}$ generation phase change memory technology. In: International Electron Devices Meeting Technical Digest, 2009. 113-116

14 Zuo Q Y. Research on a memory device with resistive switching mechanism for cross array (in Chinese). Master Dissertation. Beijing: Institute of Microelectronics, Chinese Academy of Sciences, 2010 [左青云. 适用于交叉阵列的阻变机制存储器件研究. 硕士学位论 文. 北京: 中国科学院微电子研究所, 2010]

15 Lee M J, Park Y, Suh D S, et al. Two series oxide resistors applicable to high speed and high density nonvolatile memory. Adv Mater, 2007, 19: 3919-3923

16 Yang X Y. Research on resistance change mechanism and integration technology of resistive memory (in Chinese). Master Dissertation. Beijing: Institute of Microelectronics, Chinese Academy of Sciences, 2013 [杨晓一. 阻变存储器电阻转变机理与集成技术研究. 硕士 学位论文. 北京: 中国科学院微电子研究所, 2013]

17 Zhang K W. Resistor-reversible memory based on rectification characteristics (in Chinese). Master Dissertation. Beijing: Institute of Microelectronics, Chinese Academy of Sciences, 2012 [张康玮. 基于整流特性的阻变存储器. 硕士学位论文. 北京: 中国科学院微 电子研究所, 2012]

18 Golubovic D S, Miranda A H, Akil N, et al. Vertical poly-Si select pn-diodes for emerging resistive non-volatile memories. Microelectron Eng, 2007, 84: 2921-2926

19 Ahn S E, Kang B S, Kim K H, et al. Stackable all-oxide-based nonvolatile memory with $\mathrm{Al}_{2} \mathrm{O}_{3}$ antifuse and p-CuO $\mathrm{O}_{x} / \mathrm{n}-\mathrm{InZnO} \mathrm{O}_{x}$ diode IEEE Electr Device L, 2009, 30: 550-552

20 Shima H, Takano F, Muramatsu H, et al. Control of resistance switching voltages in rectifying $\mathrm{Pt} / \mathrm{TiO}_{x} / \mathrm{Pt}$ trilayer. Appl Phys Lett, 2008, 92, doi: 10.1063/1.2838350

21 Teo E Y H, Zhang C F, Lim S L, et al. An organic-based diode-memory device with rectifying property for crossbar memory array applications. IEEE Electr Device L, 2009, 30: 487-489

22 Katsia E, Huby N, Tallarida G, et al. Poly (3-hexylthiophene)/ZnO hybrid pn junctions for microelectronics applications. Appl Phys Lett, 2009, 94, doi: 10.1063/1.3114442

$23 \mathrm{Lu} \mathrm{C}$, Yu J, Chi X W, et al. Self-compliance $\mathrm{Pt} / \mathrm{HfO}_{2} / \mathrm{Ti} / \mathrm{Si}$ one-diode-one-resistor resistive random access memory device and its low temperature characteristics. Appl Phys Express, 2016, 9, doi: 10.7567/APEX.9.041501

24 Cho B, Kim T W, Song S, et al. Rewritable switching of one diode-one resistor nonvolatile organic memory devices. Adv Mater, 2009, 21: $1228-1232$

25 Shin Y C, Song J, Kim K M, et al. ( $\mathrm{InSn})_{2} \mathrm{O}_{3} / \mathrm{TiO}_{2} / \mathrm{Pt}$ schottky-type diode switch for the $\mathrm{TiO}_{2}$ resistive switching memory array. Appl Phys Lett, 2008, 92, doi: 10.1063/1.2912531

26 Lee M J, Seo S, Kim D C, et al. A low-temperature-grown oxide diode as a new switch element for high-density, nonvolatile memories. Adv Mater, 2007, 19: 73-76

27 Lee W Y, Mauri D, Hwang C. High-current-density $\mathrm{ITO}_{x} / \mathrm{NiO}_{x}$ thin-film diodes. Appl Phys Lett, 1998, 72: 1584-1586

28 Kang B S, Ahn S E, Lee M J, et al. High-current-density $\mathrm{CuO}_{x} / \mathrm{InZnO}_{x}$ thin-film diodes for cross-point memory applications. Adv Mater, 2008, 20: 3066-3069

29 Tallarida G, Huby N, Kutrzeba-Kotowska B, et al. Low temperature rectifying junctions for crossbar non-volatile memory devices. In: International Electron Devices Meeting International Memory Workshop, 2009, doi: 10.1109/IMW.2009.5090598

30 Huang J J, Kuo C W, Chang W C, et al. Transition of stable rectification to resistive-switching in Ti/TiO $/ 2$ Pt oxide diode. Appl Phys Lett, 2010, 96, doi: 10.1063/1.3457866 


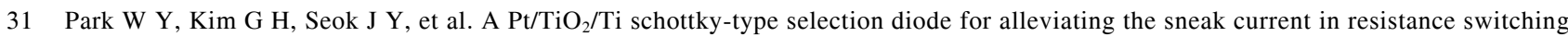
memory arrays. Nanotechnology, 2010, 21, doi: 10.1088/0957-4484/21/19/195201

32 Katsia E, Tallarida G, Kutrzeba-Kotowska B, et al. Integration of organic based schottky junctions into crossbar arrays by standard UV lithography. Org Electron, 2008, 9: 1044-1050

33 Li Y T, Liu Q, Long S B, et al. Bipolar one diode-one resistor integration for high-density resistive memory applications. Nanoscale, 2013, 5: 4785-4789

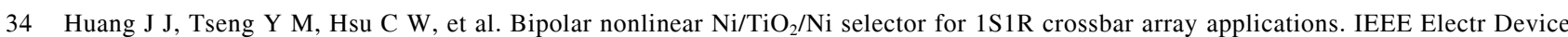
L, 2011, 9: 1427-1429

35 Luo Q, Xu X X, Liu H T, et al. Cu BEOL compatible selector with high selectivity $\left(>10^{7}\right)$, extremely low off-current $(\sim \mathrm{pA})$ and high endurance $\left(>10^{10}\right)$. In: International Electron Devices Meeting Technical Digest, 2015, 15: 253-256

36 Lee W, Park J, Kim S, et al. High current density and nonlinearity combination of selection device based on $\mathrm{TaO}_{x} / \mathrm{TiO}_{2} / \mathrm{TaO}_{x}$ structure for one selector-one resistor arrays. Acs Nano, 2012, 6: 8166-8172

37 Midya R, Wang Z R, Zhang J M, et al. Anatomy of Ag/Hafnia-based selectors with $10^{10}$ nonlinearity. Adv Mater, 2017, 29: 10.1002/adma.201604457

38 Luo Q, Xu X X, Lv H B, et al. Highly uniform and nonlinear selection device based on trapezoidal band structure for high density nano-crossbar memory array. Nano Res, 2017, 10: 3295-3302

39 Zhao X L, Ma J, Xiao X H, et al. Breaking the current-retention dilemma in cation-based resistive switching devices utilizing graphene with controlled defects. Adv Mater, 2018, 10: 10.1002/adma.201705193

40 Yang H X, Li M H, He W, et al. Novel selector for high density non-volatile memory with ultra-low holding voltage and 10(7) on/off ratio. In: Symposium on VLSI Technology, 2015. T130-T131

41 Jo S H, Kumar T, Narayanan S, et al. 3D-stackable crossbar resistive memory based on field assisted superlinear threshold (FAST) selector. In: International Electron Devices Meeting Technical Digest, 2014. 14: 160-163

42 Kim S, Kim Y B, Kim K M, et al. Performance of threshold switching in chalcogenide glass for 3D stackable selector. In: Symposium on VLSI Technology. 2013. T240-T241

43 Kim W G, Lee H M, Kim B Y, et al. $\mathrm{NbO}_{2}$-based low power and cost effective 1S1R switching for high density cross point ReRAM Application. In: Symposium on VLSI Technology, 2014. 1-2

44 Linn E, Rosezin R, Kügeler C, et al. Complementary resistive switches for passive nanocrossbar memories. Nat Mater, 2010, 9: 403-406

45 Lee M J, Lee C B, Lee D, et al. A fast, high-endurance and scalable non-volatile memory device made from asymmetric $\mathrm{Ta}_{2} \mathrm{O}_{5-x} / \mathrm{TaO}_{2-x}$ bilayer structures. Nat Mater, 2011, 10: 625-630

46 Breuer T, Kim W, Menzel S, et al. Low-current and high-endurance logic operations in $4 \mathrm{~F}^{2}$-compatible $\mathrm{TaO}_{x}$-based complementary resistive switches. In: International Electron Devices Meeting Silicon Nanoelectronics Workshop, 2014, 10: 10.1109/SNW.2014.7348558

47 Shi K X, Wang Z Q, Xu H Y, et al. Complementary resistive switching observed in Graphene oxide-based memory device. IEEE Electr Device L, 2018, 39: 488-491

48 Chang S H, Lee S B, Jeon D Y, et al. Oxide double-layer nanocrossbar for ultrahigh-density bipolar resistive memory. Adv Mater, 2011, 23: 4063-4067

49 Tran X A, Zhu W G, Gao B, et al. A self-rectifying HfO $_{x}$-based unipolar RRAM with NiSi electrode. IEEE Electr Device L, 2012, 4: 585-587

50 Tran X A, Zhu W, Liu W J, et al. A self-rectifying AlO $y$ bipolar RRAM with sub-50- $\mu$ A set/reset current for cross-bar architecture. IEEE Electr Device L, 2012, 10: 1402-1404

51 Li Y T, Jiang X Y, Tao C L, et al. A self-rectifying bipolar RRAM device based on Ni/ $\mathrm{HfO}_{2} / \mathrm{n}^{+}-\mathrm{Si}$ structure. Mod Phys Lett B, 2014, 28: $10.1142 / \mathrm{S} 0217984914500304$

52 Lv H B, Li Y T, Liu Q, et al. Self-rectifying resistive-switching device with a-Si/WO 3 bilayer. IEEE Electr Device L, 2013, 34: 229-231

53 Zuo Q Y, Long S B, Yang S Q, et al. $\mathrm{ZrO}_{2}$-based memory cell with a self-rectifying effect for crossbar WORM memory application. IEEE Electr Device L, 2010, 31: 344-346

54 Tran X A, Zhu W, Liu W J, et al. Self-selection unipolar HfO $_{x}$-based RRAM. IEEE T Electron Dev, 2013, 60: 391-395

55 Chen Y C, Chen C F, Chen C T, et al. An access-transistor-free (0T/lR) non-volatile resistance random access memory (RRAM) using a novel threshold switching, self-rectifying chalcogenide device. In: International Electron Devices Meeting Technical Digest, 2003. 905-908

56 Zuo Q Y, Long S B, Liu Q, et al. Self-rectifying effect in gold nanocrystal-embedded zirconium oxide resistive memory. J Appl Phys, 2009, 106, doi: 10.1063/1.3236632

57 Liang X F, Chen Y, Yang B, et al. A nanoscale nonvolatile memory device made from $\mathrm{RbAg}_{4} \mathrm{I}_{5}$ solid electrolyte grown on a Si substrate. Microelectron Eng, 2008, 85: 1736-1738

58 Dong Y J, Yu G H, Michael M C, et al. Si/a-Si core/shell nanowires as nonvolatile crossbar switches. Nano Lett, 2008, 8: 386-391

59 Jo S H, Lu W. CMOS compatible nanoscale nonvolatile resistance switching memory. Nano Lett, 2008, 8: 392-397

60 Kim K H, Jo S H, Gaba S, et al. Nanoscale resistive memory with intrinsic diode characteristics and long endurance. Appl Phys Lett, 2010, 96, doi: 10.1063/1.3294625 
Summary for “阻变存储器无源高密度交叉阵列研究进展”

\title{
An overview of resistive random access memory based high-density crossbar array
}

\author{
Xiaoyan $\mathrm{Li}^{1,2}$, Yingtao $\mathrm{Li}^{2 *}$, Xiaoping Gao ${ }^{1}$, Chuanbing $\mathrm{Chen}^{2} \&$ Genliang $\mathrm{Han}^{1 *}$ \\ ${ }^{1}$ Key Laboratory of Sensor and Sensing Technology, Gansu Province, Lanzhou 730000, China; \\ ${ }^{2}$ School of Physical Science and Technology, Lanzhou University, Lanzhou 730000, China \\ *Corresponding authors, E-mail: li_yt06@1zu.edu.cn; genlianghan@163.com
}

In the global era of knowledge economy and electronic information, semiconductor memories play a crucial role in the storage of the massive information. In order to achieve a higher integration density, the microelectronics process node is pushed forward to the next generation according to the Moore's Law. However, the current mainstream nonvolatile memory technology based on charge storage, such as flash memory, is rapidly running into its physical limit due to the tradeoffs between the high speed, long time retention and low power operation. Therefore, several new types of nonvolatile memories based on other storage concepts have been intensively investigated to replace Flash. Resistive random access memory (RRAM) device, which is based on resistance change modulated by electrical stimulus, has been considered as one of the most promising candidate for next-generation nonvolatile memory due to its potential advantages for simple structure, fast switching speed, excellent scalability, three-dimensional (3D) stackable integration, and good compatibility with the current complementary metal oxide semiconductor (CMOS) technology. However, a crossbar array consisting of only RRAM cell suffers unavoidable cross-talk interference due to leakage current paths through neighboring unselected cells with low resistances, leading to a misreading problem, the biggest hindrance for the high-density memory application. It can be effectively tackled by the addition of necessary nonlinearity to the RRAM by integrating a highly nonlinear and bidirectional selector device. In this paper, we give an overview on the familiar architectures to diminish the sneak current in crossbar array, including 1D1R (one diode one resistor), 1S1R (one selector one resistor), 1CRS1R (one CRS device one resistor, complementary resistive switch (CRS) devices forming by two back-to-back connected memory cells) and $1 \mathrm{R}$ (one resistor) with self-rectifying effect. In the meantime, we discuss the research trends and the challenges of high-density storage based on RRAM passive cross-array. Finally, future research direction and prospects, as well as main challenges awaiting RRAM high-density memory are given. The application of diodes with large forward current density, high rectification ratio, low preparation temperature and easy 3D integration are the key to achieve high density in 1D1R structure integration. It is of great significance to find materials with better performance and applicability, and to carry out theoretical research on the integrated applications of $1 \mathrm{~S} 1 \mathrm{R}$ and $1 \mathrm{CRS} 1 \mathrm{R}$ structures. Since the $1 \mathrm{R}$ with self-rectifying effect is simpler and more cost-effective than other integrated structures, further research to clarify intrinsic physical mechanism of $1 \mathrm{R}$, and develop high-performance self-rectifying RRAM devices with large rectification ratio, high current density, uniformity, stability, and reliability are the significant processes to the achievement of RRAM high-density storage applications.

resistive random access memory, high density, passive crossbar array, cross-talk

doi: 10.1360/N972018-00164 